\title{
Correction to: PRSS8 suppresses colorectal carcinogenesis and metastasis
}

Yonghua Bao · Yongchen Guo • Yiqiong Yang • Xiaonan Wei · Shanshan Zhang • Yongmeng Zhang • Kai Li • Ming Yuan · Dongli Guo • Virgilia Macias · Xiangdong Zhu • Wei Zhang • Wancai Yang

Published online: 22 February 2021

(c) The Author(s), under exclusive licence to Springer Nature Limited 2021

Correction to: Oncogene

https://doi.org/10.1038/s41388-018-0453-3
In the original published version Fig. $4 \mathrm{c}$ and Fig. $5 \mathrm{~d}$ are incorrect. The correct figures are given below.

The original article was corrected. 
C

C-1

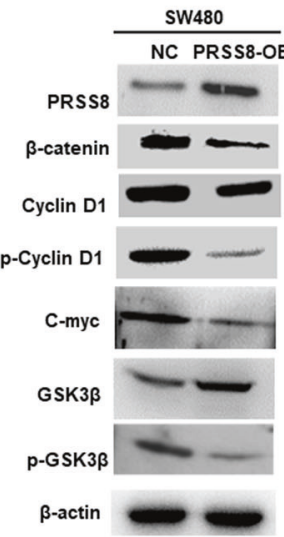

HCT116

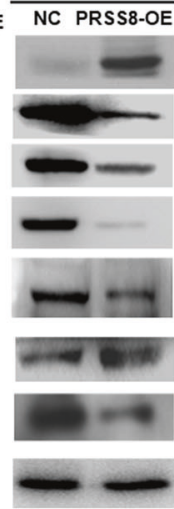

C-2

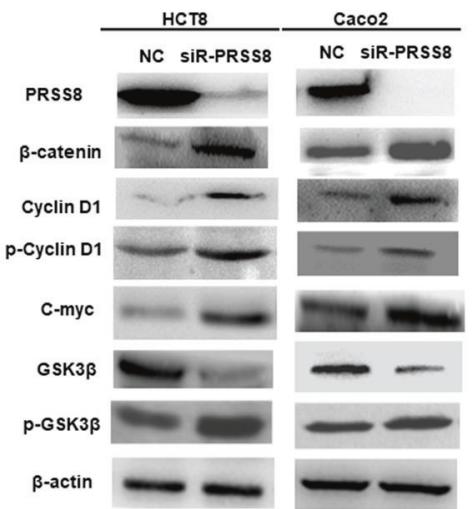

D

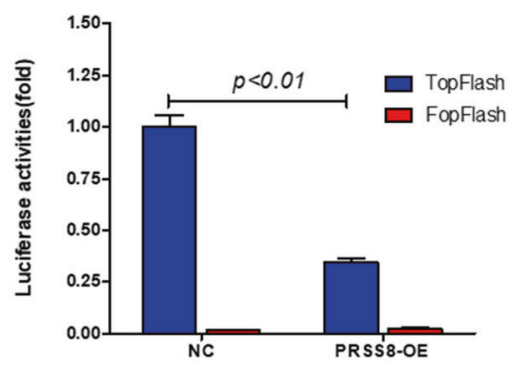

$\mathbf{F}$

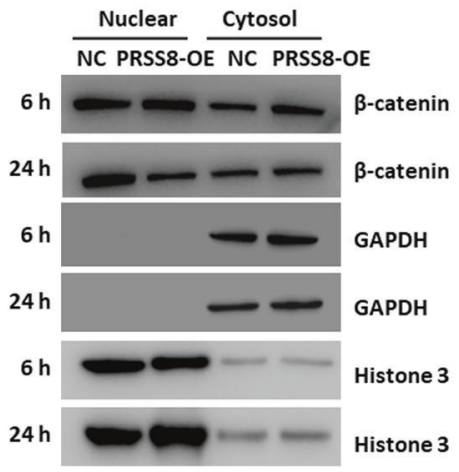

G

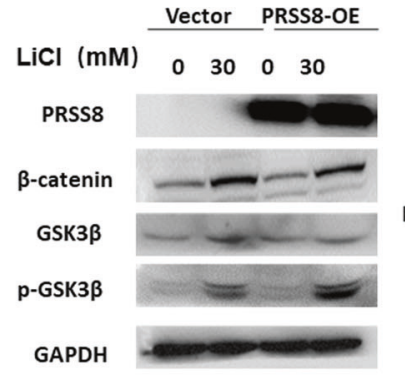

$\mathrm{E}$

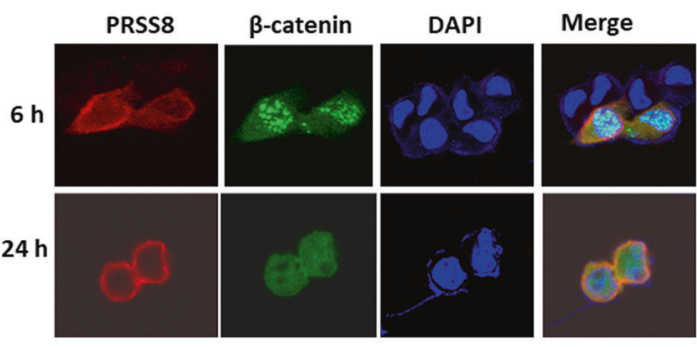

H

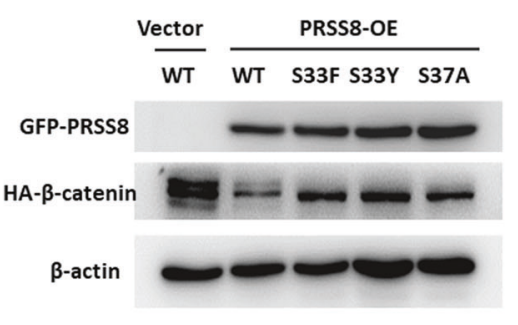

Fig. 4 Molecular mechanisms of PRSS8-mediated suppression of carcinogenesis and metastasis, and crosstalk between PRSS8 and the Wnt/ $\beta$-catenin pathway. a Gene profile on HCt166 cells and gene set enrichment analysis (GSEA) showed that PRSS8 expression is associated with PI3K-AKT, Wnt//-catenin, epithelial-mesenchymal transition (EMT) and stem cell signaling pathways. b RNA sequence on mouse intestinal epithelial cells from Prss8flfl, Cre + and wild-type mice and GSEA showed significant changes in certain major signaling pathways. $\mathbf{c}$ PRSS8 affected $\beta$-catenin signaling at the protein level in SW480, HCT116, HCT8 and Caco2 colon cancer cells transiently transfected with vector only (negative control, NC), PRSS8 over-expression plasmid (PRSS8-OE), or siRNA targeting PRSS8 (siRNA-PRSS8). d Increased PRSS8 expression suppressed $\beta$-catenin-TCF4 luciferase activity, as assayed by determining TopFlash activity. FopFlash was used as a control. e, f PRSS8 affected $\beta$-catenin cyto-plasmic/nuclear translocation, as determined by immunofluorescence staining and cellular fragment immunoblotting (GAPDH was used as a cytoplasmic loading control, and Histone 3 was used as a nuclear loading control). g LiCl caused GSk3bta phosphorylation and $\beta$-catenin upregulation (lane 2 vs lane 1 ), and the phosphorylated GSK3 $\beta$ (p-GSk3 $\beta$ ) affected PRSS8-mediated $\beta$-catenin degradation (lane 4 vs lane 3), in HCT116 cells. h Mutation of GSK3 $\beta$-mediated phosphorylation sites on the $\beta$-catenin protein attenuated PRSS8-induced degradation of the $\beta$-catenin protein in HEK293 cells. (WT, wild-type $\beta$-catenin; S33F, serine 33 to phenylalanine 33; S33Y, serine 33 to tyrosine 33; S37A, serine 37 to alanine 37 ). 
A
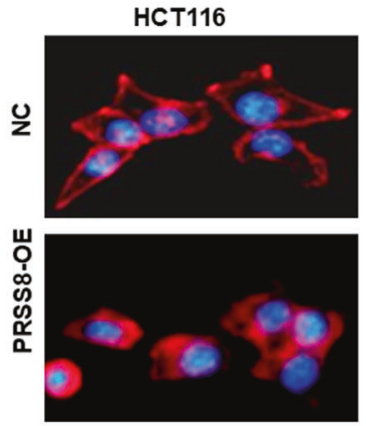

C
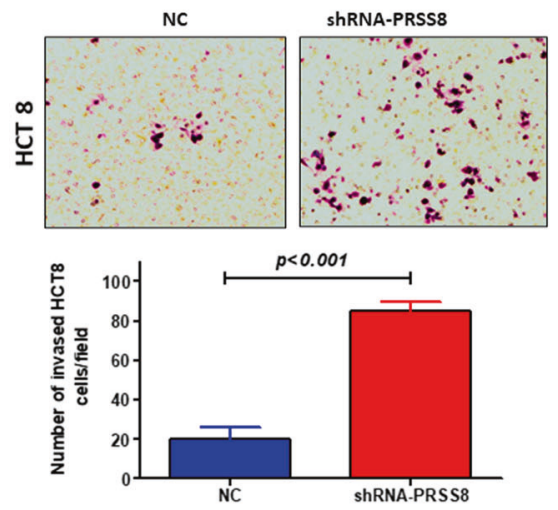

D
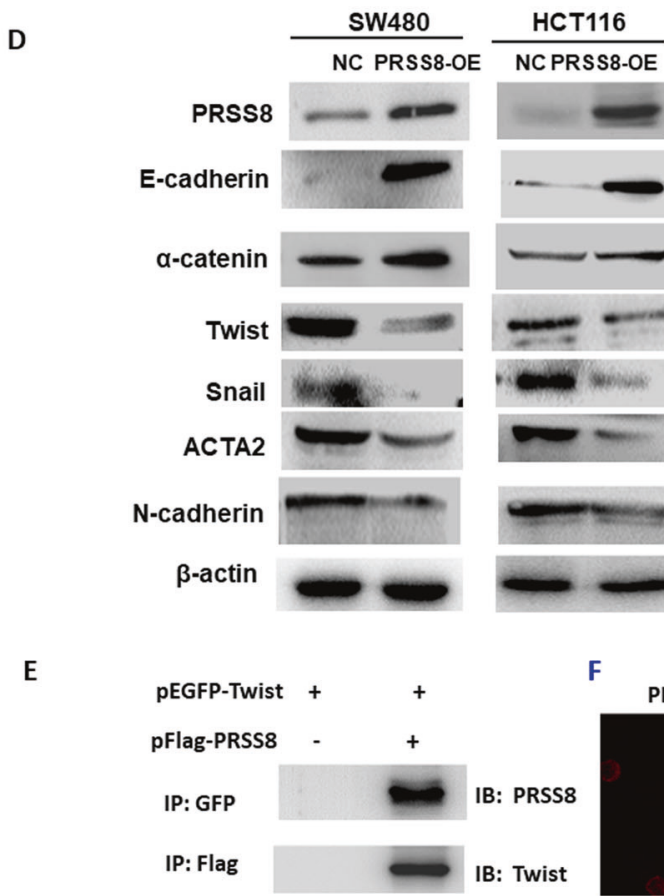

$\mathbf{F}$

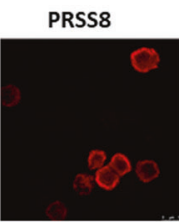

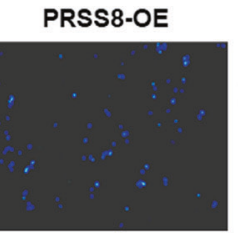
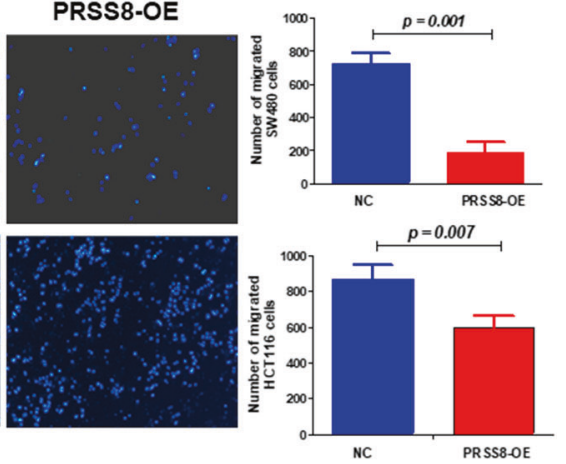
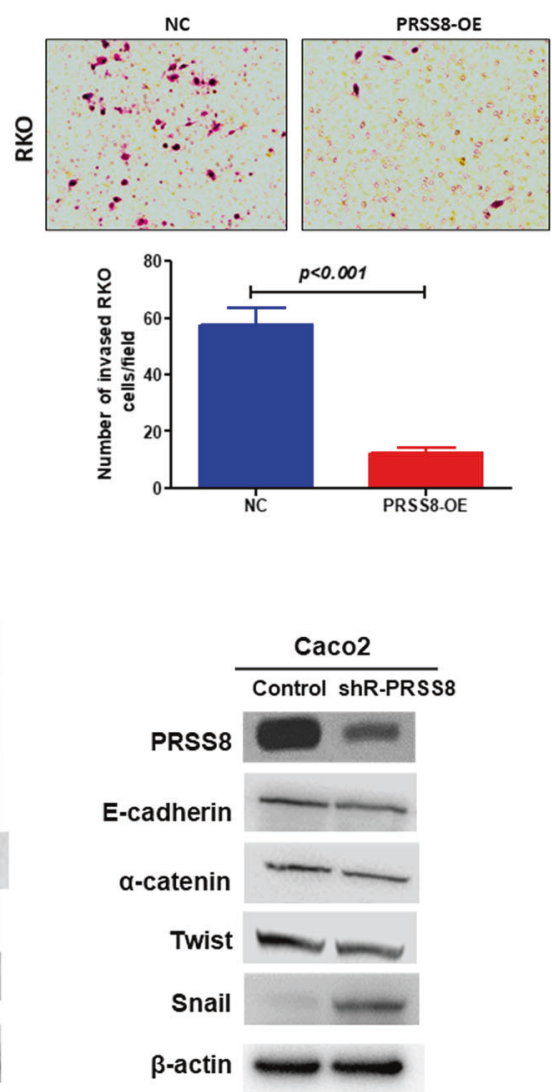

Fig. 5 PRSS8 altered cancer cell morphology and suppressed the epithelial-mesenchymal transition pathway. a PRSS8 overexpression induced cytoskeletal changes, converting HCT116 cells from an invasive, spindle-like morphology to a less invasive, rounded pheno-type. b Increased PRSS8 expression inhibited migration of SW480 and HCT116 cancer cells. c Knockdown of PRSS8 expression promoted invasion of HCT8 cancer cells, and increased PRSS8 expression suppressed invasion of RKO cancer cells. d PRSS8 expression level saffected EMT-related protein changes. e Co-immunoprecipitation assay showed binding between PRSS8 and TWIST proteins. f PRSS8 and Twist were overlapped in subcellular localization in HCT116 cells by immunofluorescence staining. (NC negative control; shRNA-PRSS8, knockdown of PRSS8 expression by a virus-based short hairpin RNA vector targeting the PRSS8 gene; PRSS8-OE,increased expression of PRSS8 induced by a virus-based expression plasmid. IB immunoblotting, IP co-immunoprecipitation). 